Article

\title{
On a New Class of Fractional Difference-Sum Operators with Discrete Mittag-Leffler Kernels
}

\author{
Thabet Abdeljawad ${ }^{1(1)}$ and Arran Fernandez $2,3, *$ (D) \\ 1 Department of Mathematics and General Sciences, Prince Sultan University, P.O. Box 66833, Riyadh 11586, \\ Saudi Arabia \\ 2 Department of Applied Mathematics and Theoretical Physics, University of Cambridge, Wilberforce Road, \\ Cambridge, Cambridge CB3 0WA, UK \\ 3 Department of Mathematics, Faculty of Arts and Sciences, Eastern Mediterranean University, 99628 Famagusta, \\ North Cyprus, via Mersin 10, Turkey \\ * Correspondence: arran.fernandez@emu.edu.tr
}

Received:18 July 2019; Accepted: 19 August 2019; Published: 22 August 2019

check for updates

\begin{abstract}
We formulate a new class of fractional difference and sum operators, study their fundamental properties, and find their discrete Laplace transforms. The method depends on iterating the fractional sum operators corresponding to fractional differences with discrete Mittag-Leffler kernels. The iteration process depends on the binomial theorem. We note in particular the fact that the iterated fractional sums have a certain semigroup property, and hence, the new introduced iterated fractional difference-sum operators have this semigroup property as well.
\end{abstract}

Keywords: discrete fractional calculus; Atangana-Baleanu fractional differences and sums; discrete Mittag-Leffler function; discrete nabla Laplace transform; binomial theorem; iterated process; discrete Dirac delta function

MSC: 39A12; 34A25; 26A33

\section{Introduction}

Discrete fractional calculus is an important emerging branch of analysis [1-3], which has been very useful in the analysis of discrete systems with non-local effects. The solution of discrete fractional differential equations and discrete boundary value problems has discovered many applications, and so, this is an important field to develop in terms of mathematical theory.

There are many different types of fractional calculus, which can be defined in both the continuous and discrete contexts. The point of such an exercise is to discover new ways of modelling various fractional systems, and to create new frameworks, which can then be used in a number of applications [4,5], including in health sciences [6,7], control theory [8,9], and bioengineering [10,11]. It is important to continue developing these new models: both from the point of view of increasing mathematical understanding for its own sake and also from the point of view of the applications just described, since a better understanding of the underlying mathematics will enable a better analysis of the physical models they describe.

In the last two years, some important new definitions of fractional calculus have been formulated with exponential and Mittag-Leffler kernels [12,13], and their properties have been explored in a number of papers $[14,15]$. Furthermore, discrete fractional calculus has been theoretically developed more by formulating and analysing discrete versions of these fractional operators $[16,17]$. The idea of our approach in this article depends on iterating the fractional sums corresponding to fractional operators with discrete Mittag-Leffler kernels, extending and generalising these operators in such 
a way as to recover certain desirable traits such as a semigroup property. The idea of considering iterations of functional operators to get fractional ones is not new (indeed, it is the very basis of fractional calculus itself), but it has recently been explored as a way of adding further fractionalisation to operators that are already considered as fractional $[18,19]$. The present work can be seen as an extension of these projects into the discrete context. The advantage of the discrete operators compared to the existing ones is the same as in any branch of discrete calculus: it is useful to have definitions in both discrete and continuous contexts, since modelling different processes in the real world requires both discrete and continuous models $[2,3,20,21]$.

The structure of this work is as follows. In Section 2, we review some basic concepts about discrete fractional calculus in the frame of nabla difference analysis, including the well-known Atangana-Baleanu $(\mathrm{AB})$ model of discrete fractional calculus and its fundamental properties. In Section 3, we define our new family of operators and analyse them, proving some essential facts about them. In Section 4, we consider some fractional difference equations in this new model. Finally, in Section 5, we conclude the paper.

\section{Basics of Nabla Discrete Fractional Calculus}

Definition 1. (i) For any $l \in \mathbb{N}$ and any number $z$, the l rising factorial of $z$ is:

$$
z^{\bar{l}}=\prod_{i=0}^{l-1}(z+i), \quad z^{\overline{0}}=1 .
$$

(ii) For any $\mu \in \mathbb{R}$, the $\mu$ rising function is:

$$
z^{\bar{\mu}}=\frac{\Gamma(z+\mu)}{\Gamma(z)}, \quad z \in \mathbb{R} \backslash\{\ldots,-2,-1,0\}, 0^{\bar{\mu}}=0,
$$

where $\Gamma$ denotes the Euler gamma function.

The following fact is straightforward to prove:

$$
\nabla\left(z^{\bar{\mu}}\right)=\mu z^{\overline{\mu-1}}
$$

and this means $z^{\bar{\mu}}$ is an increasing function on $\mathbb{N}_{0}$.

Definition 2 (Nabla fractional sums; see [22,23]). Define the operator $\rho(t)=t-1$, which is called the backwards jump. For any function $f: \mathbb{N}_{a}:=\{a, a+1, a+2, ..\} \rightarrow \mathbb{R}$, the nabla fractional sum of order $\mu>0$ and of the left type starting from $a$ is defined by:

$$
{ }_{a} \nabla^{-\mu} f(z)=\frac{1}{\Gamma(\mu)} \sum_{s=a+1}^{z}(z-\rho(s))^{\overline{\mu-1}} f(s), \quad z \in \mathbb{N}_{a+1} .
$$

Similarly, for a function $f:{ }_{b} \mathbb{N}:=\{b, b-1, b-2, ..\} \rightarrow \mathbb{R}$, the nabla fractional sum of order $\mu>0$ and of right type finishing at $b$ is defined by:

$$
\begin{aligned}
\nabla_{b}^{-\mu} f(z) & =\frac{1}{\Gamma(\mu)} \sum_{s=z}^{b-1}(s-\rho(z))^{\overline{\mu-1}} f(s) \\
& =\frac{1}{\Gamma(\mu)} \sum_{s=z}^{b-1}(\sigma(s)-z)^{\overline{\mu-1}} f(s), \quad z \in_{b-1} \mathbb{N} .
\end{aligned}
$$


Lemma 1 ([23,24]). Let $\alpha>0, \beta>-1, h>0$. Then, we have the following identities:

$$
\begin{aligned}
{ }_{a} \nabla^{-\alpha}(t-a)^{\bar{\beta}} & =\frac{\Gamma(\beta+1)}{\Gamma(\beta+1+\alpha)}(t-a)^{\overline{\alpha+\beta}} \\
\nabla_{b}^{-\alpha}(b-t)^{\bar{\beta}} & =\frac{\Gamma(\beta+1)}{\Gamma(\beta+1+\alpha)}(b-t)^{\overline{\alpha+\beta}}
\end{aligned}
$$

From [2,23], we recall that the left and right nabla fractional sums satisfy the following semigroup property:

$$
\begin{aligned}
{ }_{a} \nabla^{-\alpha}{ }_{a} \nabla^{-\mu} f(v) & ={ }_{a} \nabla^{-(\alpha+\mu)} f(v), \\
\nabla_{b}^{-\alpha} \nabla_{b}^{-\mu} f(v) & =\nabla_{b}^{-(\alpha+\mu)} f(v)
\end{aligned}
$$

Definition 3 (Nabla discrete Laplace transforms; see [17]). The nabla discrete Laplace transform $\mathcal{K}=\mathcal{K}_{0}$, applied to a function $f$ defined on $\mathbb{N}_{0}$, is defined by $\mathcal{K} f(z)=\sum_{t=1}^{\infty}(1-z)^{t-1} f(t)$.

More generally, for any $a$, if $f$ is a function on $\mathbb{N}_{a}$, the nabla discrete Laplace transform $\mathcal{K}_{a}$ is defined by $\mathcal{K}_{a} f(z)=\sum_{t=a+1}^{\infty}(1-z)^{t-a-1} f(t)$.

Lemma 2. For any $\mu \in \mathbb{R} \backslash\{\ldots,-2,-1,0\}$, we have the following results on nabla discrete Laplace transforms.

(i) $\mathcal{K}\left(t^{\overline{\mu-1}}\right)(z)=\frac{\Gamma(\mu)}{z^{\mu}},|1-z|<1$,

(ii) $\mathcal{K}\left(t^{\overline{\mu-1}} b^{-t}\right)(z)=\frac{b^{\mu-1} \Gamma(\mu)}{(z+b-1)^{\mu}},|1-z|<b$.

Proof. See [25].

Remark 1. We can extend (i) of Lemma 2 to the more general statement that:

$$
\left(\mathcal{K}_{a}(t-a)^{\overline{\mu-1}}\right)(s)=\frac{\Gamma(\mu)}{s^{\mu}} .
$$

Definition 4. [Nabla discrete Mittag-Leffler; see $[17,22,23,26]$ ] For $\lambda \in \mathbb{R}$ with $|\lambda|<1$ and $\alpha, \beta, \rho, v \in \mathbb{C}$ with $\operatorname{Re}(\alpha)>0$, the nabla discrete Mittag-Leffler functions with one, two, and three parameters are defined respectively by:

$$
\begin{aligned}
E_{\bar{\alpha}}(\lambda, v) & =\sum_{k=0}^{\infty} \lambda^{k} \frac{v^{\overline{k \alpha}}}{\Gamma(\alpha k+1)} ; \\
E_{\overline{\alpha, \beta}}(\lambda, v) & =\sum_{k=0}^{\infty} \lambda^{k} \frac{v^{\overline{k \alpha+\beta-1}}}{\Gamma(\alpha k+\beta)} ; \\
E_{\alpha, \beta}^{\rho}(\lambda, v) & =\sum_{k=0}^{\infty}(\rho)_{k} \frac{v^{k}}{k ! \Gamma(\alpha k+\beta)} .
\end{aligned}
$$
case [27].

Note that we have $E_{\bar{\alpha}}(\lambda, v)=E_{\overline{\alpha, 1}}(\lambda, v)$ and $E_{\overline{\alpha, \beta}}(\lambda, v)=E_{\overline{\alpha, \beta}}^{1}(\lambda, v)$, just as in the continuous Proposition 1. For any $\lambda, \alpha, \beta, \rho, v \in \mathbb{C}$ as in Definition 4 and $\gamma \in \mathbb{C}$ with $\operatorname{Re}(\gamma)>0$, we have the following difference and summation properties of discrete Mittag-Leffler functions. 


$$
\begin{aligned}
\nabla_{v} E_{\bar{\alpha}}(\lambda, v) & =\lambda E_{\overline{\alpha, \alpha}}(\lambda, v) ; \\
\nabla_{v} E_{\overline{\alpha, \beta}}^{\rho}(\lambda, v) & =E_{\overline{\alpha, \beta-1}}^{\rho}(\lambda, v) ; \\
\sum_{t=a+1}^{v} E_{\overline{\alpha, \beta}}(\lambda, t-a) & =E_{\overline{\alpha, \beta+1}}(\lambda, v-a) ; \\
{ }_{a} \nabla^{-\gamma} E_{\overline{\alpha, \beta}}^{\rho}(\lambda, v-a) & =E_{\overline{\alpha, \beta+\gamma}}^{\rho}(\lambda, v-a) .
\end{aligned}
$$

Proof. The proof is straightforward (see [17]). In the proof of the last part, the assertion [2,22-24] that:

$$
{ }_{a} \nabla^{-\gamma}(t-a)^{\overline{v-1}}=\frac{\Gamma(v)}{\Gamma(v+\gamma)}(t-a)^{\overline{\gamma+v-1}}
$$

has been used. Furthermore, notice that the second part is the particular case $\gamma=-1$ of the last part.

The remainder of this section is dedicated to summarising some known results about discrete Laplace transforms for Mittag-Leffler functions and functions of the convolution type. More details can be found in [26].

Definition 5 (Nabla discrete convolutions; see [2,26]). Let $a \in \mathbb{R}$, and consider two functions $f, g: \mathbb{N}_{a} \rightarrow \mathbb{R}$. Their nabla discrete convolution is:

$$
(f * g)(v)=\sum_{s=a+1}^{v} g(v-\rho(s)+a) f(s) .
$$

Proposition 2 ([2,26]). For any $a \in \mathbb{R}$ and functions $f, g$ defined on $\mathbb{N}_{a}$, we have the following convolution property of Laplace transforms in the nabla discrete context:

$$
\left(\mathcal{K}_{a}(f * g)\right)(s)=\left(\mathcal{K}_{a} f\right)(s)\left(\mathcal{K}_{a} g\right)(s)
$$

Lemma 3 ([26]). Let $a \in \mathbb{R}$, and let $f$ be a function defined on $\mathbb{N}_{a}$. Then:

$$
\left(\mathcal{K}_{a} \nabla(f(t))(s)=s\left(\mathcal{K}_{a} f\right)(s)-f(a) .\right.
$$

Lemma 4 ([25]). For any $a \in \mathbb{R}$ and $v \in \mathbb{R}^{+}$, we have:

$$
\left(\mathcal{K}_{a} a \nabla^{-v}\right) f(s)=s^{-v}\left(\mathcal{K}_{a} f\right)(s)
$$

Lemma 5 ([26]). Let $0<\alpha \leq 1, a \in \mathbb{R}$, and $f$ be a function defined on $\mathbb{N}_{a}$. Then:

$$
\left(\mathcal{K}_{a} E_{\bar{\alpha}}(\lambda, t-a)\right)(z)=\frac{z^{\alpha-1}}{z^{\alpha}-\lambda} ; \quad\left(\mathcal{K}_{a} E_{\overline{\alpha, \alpha}}(\lambda, t-a)\right)(z)=\frac{1}{z^{\alpha}-\lambda} .
$$

Discrete Atangana-Baleanu Fractional Differences

Let us review the basic theory of fractional sums and differences defined using discrete Mittag-Leffler kernels, as presented in [17] based on the original ideas in [13,27,28].

Definition 6 ([17]). Let $\alpha \in[0,1]$ and $a<b$ in $\mathbb{R}$. For a function $f$ defined on $\mathbb{N}_{a}$, its nabla discrete ABleft fractional difference is defined as Caputo type by: 


$$
\left({ }_{a}^{A B C} \nabla^{\alpha} f\right)(t)=\frac{B(\alpha)}{1-\alpha} \sum_{s=a+1}^{t} \nabla_{s} f(s) E_{\bar{\alpha}}\left(\frac{-\alpha}{1-\alpha}, t-\rho(s)\right),
$$

and as Riemann-Liouville type by:

$$
\left({ }_{a}^{A B R} \nabla^{\alpha} f\right)(t)=\frac{B(\alpha)}{1-\alpha} \nabla_{t} \sum_{s=a+1}^{t} f(s) E_{\bar{\alpha}}\left(\frac{-\alpha}{1-\alpha}, t-\rho(s)\right),
$$

where in both cases, $B(\alpha)$ is an arbitrary multiplier function satisfying $B(0)=B(1)=1$, which may be assumed [14] to be real and positive. Similarly, for a function $f$ defined on ${ }_{b} \mathbb{N}$, its nabla discrete AB right fractional difference is defined as Caputo type by:

$$
\left({ }^{A B C} \nabla_{b}^{\alpha} f\right)(t)=\frac{B(\alpha)}{1-\alpha} \sum_{s=t}^{b-1}\left(-\Delta_{s} f\right)(s) E_{\bar{\alpha}}\left(\frac{-\alpha}{1-\alpha}, s-\rho(t)\right) .
$$

and as Riemann-Liouville type by:

$$
\left({ }^{A B R} \nabla_{b}^{\alpha} f\right)(t)=\frac{B(\alpha)}{1-\alpha}\left(-\Delta_{t}\right) \sum_{s=t}^{b-1} f(s) E_{\bar{\alpha}}\left(\frac{-\alpha}{1-\alpha}, s-\rho(t)\right) .
$$

The notations $A B R$ and $A B C$ are used to denote $A B$ fractional differences of Riemann-Liouville and Caputo type, respectively.

Note that since the discrete Mittag-Leffler kernel (8) converges for $|\lambda|<1$, and in this case, $|\lambda|=\frac{\alpha}{1-\alpha}$; the kernels in all four of the above definitions are convergent for $0<\alpha<\frac{1}{2}$.

We now define the fractional sums corresponding to the fractional difference defined in Definition 6. Again, this is analogous to the definition found in [13] for the continuous case.

Definition 7 ([17]). For $0<\alpha<1$ and a function $f$ defined on $\mathbb{N}_{a}$, the left fractional sum of $A B$ type is:

$$
\left({ }_{a}^{A B} \nabla^{-\alpha} f\right)(t)=\frac{1-\alpha}{B(\alpha)} f(t)+\frac{\alpha}{B(\alpha)}\left({ }_{a} \nabla^{-\alpha} f\right)(t) .
$$

Similarly, for a function $f$ defined on ${ }_{b} \mathbb{N}$, the right fractional sum of $A B$ type is:

$$
\left({ }^{A B} \nabla_{b}^{-\alpha} f\right)(t)=\frac{1-\alpha}{B(\alpha)} f(t)+\frac{\alpha}{B(\alpha)}\left(\nabla_{b}^{-\alpha} f\right)(t) .
$$

Theorem 1. For any $\alpha \in\left(0, \frac{1}{2}\right)$ and any function $f$ defined on $\mathbb{N}_{a} \cap{ }_{b} \mathbb{N}$, we have the following relations between the AB fractional differences of Caputo and Riemann-Liouville type and the associated AB fractional sum.

$$
\begin{gathered}
\left({ }_{a}^{A B R} \nabla_{a}^{\alpha}{ }_{a}^{A B} \nabla^{-\alpha} f\right)(t)=f(t) ; \\
\left({ }_{a}^{A B} \nabla^{-\alpha}{ }_{a}^{A B R} \nabla^{\alpha} f\right)(t)=f(t) ; \\
\left({ }^{A B R} \nabla_{b}^{\alpha}{ }^{A B} \nabla_{b}^{-\alpha} f\right)(t)=f(t) ; \\
\left({ }^{A B} \nabla_{b}^{-\alpha} A B R \nabla_{b}^{\alpha} f\right)(t)=f(t) ; \\
\left({ }_{a}^{A B C} \nabla^{\alpha} f\right)(t)=\left({ }_{a}^{A B C} \nabla^{\alpha} f\right)(t)-f(a) \frac{B(\alpha)}{1-\alpha} E_{\bar{\alpha}}(\lambda, t-a) ; \\
(t)=\left({ }_{b}^{A B R} \nabla_{b}^{\alpha} f\right)(t)-f(b) \frac{B(\alpha)}{1-\alpha} E_{\bar{\alpha}}(\lambda, b-t) .
\end{gathered}
$$


Proof. See [17].

The following lemma, the discrete analogue of a result proven for the continuous $\mathrm{AB}$ model in [14], is essential to proceed in confirming our representations.

Lemma 6 ([16]). For any $0<\alpha<\frac{1}{2}$, with $\lambda:=\frac{-\alpha}{1-\alpha}$ and $f$ being a function defined on $\mathbb{N}_{a}$, we have:

$$
\left({ }_{a}^{A B R} \nabla^{\alpha} f\right)(t)=\frac{B(\alpha)}{1-\alpha}\left[f(t)+\sum_{k=1}^{\infty} \lambda^{k}\left({ }_{a} \nabla^{-\alpha k} f\right)(t)\right] .
$$

\section{Iterated AB Fractional Difference-Sum Operators}

\subsection{Definitions}

In this section, by iterating the $A B$ fractional sums, we shall formulate a new class of fractional difference-sum operators, which has a semigroup property in one of its two parameters. These operators are the discrete analogue of the iterated $A B$ differintegrals defined in [18].

Consider the $\mathrm{AB}$ left fractional sum defined in (16). If we iterate this operator $n$ times using the binomial theorem and make use of the semigroup property (6) and the fact that ${ }_{a} \nabla^{-0} f(t)=f(t)$, then we have:

$$
\begin{aligned}
\left(\begin{array}{c}
A B \\
a
\end{array} \nabla^{-\alpha}\right)^{n} f(t)= & {\left[\frac{1-\alpha}{B(\alpha)}+\frac{\alpha}{B(\alpha)}{ }_{a} \nabla^{-\alpha}\right]^{n} f(t) } \\
= & \sum_{k=0}^{n} \frac{\left(\begin{array}{l}
n \\
k
\end{array}\right)(1-\alpha)^{n-k} \alpha^{k}}{B(\alpha)^{n}}{ }_{a} \nabla^{-k \alpha} f(t) \\
= & \left(\frac{1-\alpha}{B(\alpha)}\right)^{n} f(t) \\
& +\sum_{k=1}^{n} \frac{\left(\begin{array}{l}
n \\
k
\end{array}\right)(1-\alpha)^{n-k} \alpha^{k}}{B(\alpha)^{n} \Gamma(\alpha k)}\left(\sum_{s=a+1}^{t}(t-\rho(s))^{\overline{k \alpha-1}} f(s)\right) \\
= & \sum_{s=a+1}^{t} f(s)\left[\left(\frac{1-\alpha}{B(\alpha)}\right)^{n} \delta(t-\rho(s))\right. \\
& \left.+\sum_{k=1}^{n} \frac{\left(\begin{array}{l}
n \\
k
\end{array}\right)(1-\alpha)^{n-k} \alpha^{k}}{B(\alpha)^{n} \Gamma(\alpha k)}(t-\rho(s))^{\overline{k \alpha-1}}\right],
\end{aligned}
$$

where $\delta(t-\rho(s))$ is the Dirac delta function.

Now, for $\alpha \in[0,1]$, the above formulae for the iteration of $\mathrm{AB}$ discrete operators can be fractionalised by replacing the index $n$ with a general $\mu \in \mathbb{R}$ and replacing the finite sum by an infinite sum like the fractional binomial theorem. This idea is formalised in the following definition.

Definition 8. Let $\alpha \in[0,1], \mu \in \mathbb{R}$, and $f$ be a function defined on $\mathbb{N}_{a}$. The iterated left $A B$ fractional difference-sum of $f$ with order $(-\alpha, \mu)$, denoted by ${ }_{a}^{A B} \nabla^{(-\alpha, \mu)} f(t)$, is defined as:

$$
\begin{aligned}
{ }_{a}^{A B} \nabla^{(-\alpha, \mu)} f(t) & =\sum_{k=0}^{\infty} \frac{\left(\begin{array}{l}
\mu \\
k
\end{array}\right)(1-\alpha)^{\mu-k} \alpha^{k}}{B(\alpha)^{\mu}}{ }_{a} \nabla^{-k \alpha} f(t) \\
& =\sum_{s=a+1}^{t} f(s)\left[\left(\frac{1-\alpha}{B(\alpha)}\right)^{\mu} \delta(t-\rho(s))+\sum_{k=1}^{\infty} \frac{\left(\begin{array}{l}
\mu \\
k
\end{array}\right)(1-\alpha)^{\mu-k} \alpha^{k}}{B(\alpha)^{\mu} \Gamma(\alpha k)}(t-\rho(s))^{\overline{k \alpha-1}}\right] .
\end{aligned}
$$


Since $\mu$ can be negative, the left iterated $A B$ difference of order $(-\alpha,-\mu)$ can be defined in exactly the same way, i.e.,

$$
\begin{aligned}
{ }_{a}^{A B} \nabla^{(-\alpha,-\mu)} f(t) & =\sum_{k=0}^{\infty} \frac{\left(\begin{array}{c}
-\mu \\
k
\end{array}\right) B(\alpha)^{\mu} \alpha^{k}}{(1-\alpha)^{\mu+k}}{ }_{a} \nabla^{-k \alpha} f(t) \\
& =\sum_{s=a+1}^{t} f(s)\left[\left(\frac{B(\alpha)}{1-\alpha}\right)^{\mu} \delta(t-\rho(s))+\sum_{k=1}^{\infty} \frac{\left(\begin{array}{c}
-\mu \\
k
\end{array}\right) B(\alpha)^{\mu} \alpha^{k}}{(1-\alpha)^{\mu+k} \Gamma(\alpha k)}(t-\rho(s))^{\overline{k \alpha-1}}\right] .
\end{aligned}
$$

The iterated right $\mathrm{AB}$ fractional difference-sum and difference operators can be defined in an exactly analogous way to Definition 8 , namely as follows.

Definition 9. Let $\alpha \in[0,1], \mu \in \mathbb{R}$, and $f$ be a function defined on ${ }_{b} \mathbb{N}$. The iterated right $A B$ fractional difference-sum of $f$ with order $(-\alpha, \mu)$, denoted by ${ }^{A B} \nabla_{b}^{(-\alpha, \mu)} f(t)$, is defined as:

$$
\begin{aligned}
{ }^{A B} \nabla_{b}^{(-\alpha, \mu)} f(t) & =\sum_{k=0}^{\infty} \frac{\left(\begin{array}{l}
\mu \\
k
\end{array}\right)(1-\alpha)^{\mu-k} \alpha^{k}}{B(\alpha)^{\mu}} \nabla_{b}^{-k \alpha} f(t) \\
& =\sum_{s=t}^{b-1} f(s)\left[\left(\frac{1-\alpha}{B(\alpha)}\right)^{\mu} \delta(s-\rho(t))+\sum_{k=1}^{\infty} \frac{\left(\begin{array}{l}
\mu \\
k
\end{array}\right)(1-\alpha)^{\mu-k} \alpha^{k}}{B(\alpha)^{\mu} \Gamma(\alpha k)}(s-\rho(t))^{\overline{k \alpha-1}}\right] .
\end{aligned}
$$

Since $\mu$ can be negative, the right iterated $A B$ difference of order $(-\alpha,-\mu)$ can be defined in exactly the same way, i.e.,

$$
\begin{aligned}
{ }^{A B} \nabla_{b}^{(-\alpha,-\mu)} f(t) & =\sum_{k=0}^{\infty} \frac{\left(\begin{array}{c}
-\mu \\
k
\end{array}\right) B(\alpha)^{\mu} \alpha^{k}}{(1-\alpha)^{\mu+k}} \nabla_{b}^{-k \alpha} f(t) \\
& =\sum_{s=t}^{b-1} f(s)\left[\left(\frac{B(\alpha)}{1-\alpha}\right)^{\mu} \delta(s-\rho(t))+\sum_{k=1}^{\infty} \frac{\left(\begin{array}{c}
-\mu \\
k
\end{array}\right) B(\alpha)^{\mu} \alpha^{k}}{(1-\alpha)^{\mu+k} \Gamma(\alpha k)}(s-\rho(t))^{\overline{k \alpha-1}}\right] .
\end{aligned}
$$

Notice that on the time scale $\mathbb{N}$, we have $s-\rho(t)=\sigma(s)-t$, and therefore, the Dirac delta function in the sense of delta time scale analysis $\left(\delta^{\Delta}(\sigma(s)-t)\right)$ is the same as $\delta(s-\rho(t))$.

Remark 2. Below are some special cases of the above Definitions 8 and 9 , which reflect their appropriateness as definitions of a two-parameter model of fractional calculus.

- When $\mu=n \in \mathbb{N}$, we recover the expression derived above for iterating the AB fractional sum $n$ times. In particular, when $\mu=1$, we have:

$$
\begin{aligned}
& { }_{a}^{A B} \nabla^{(-\alpha, 1)} f(t)={ }_{a}^{A B} \nabla^{-\alpha} f(t) ; \\
& { }^{A B} \nabla_{b}^{(-\alpha, 1)} f(t)={ }^{A B} \nabla_{b}^{-\alpha} f(t) .
\end{aligned}
$$

- When $\alpha=0$, we recover the function $f(t)$ itself:

$$
{ }_{a}^{A B} \nabla^{(0, \mu)} f(t)={ }^{A B} \nabla_{b}^{(0, \mu)} f(t)=f(t) .
$$

- When $\mu=-1$, we have:

$$
\begin{aligned}
{ }_{a}^{A B} \nabla^{(-\alpha,-1)} f(t) & ={ }_{a}^{A B R} \nabla^{\alpha} f(t) ; \\
{ }^{A B} \nabla_{b}^{(-\alpha,-1)} f(t) & ={ }^{A B R} \nabla_{b}^{\alpha} f(t) .
\end{aligned}
$$


More generally, for $\mu=-n$, we have:

$$
\begin{aligned}
{ }_{a}^{A B} \nabla^{(-\alpha,-n)} f(t) & =\left(\underset{a}{A B R} \nabla^{\alpha}\right)^{n} f(t) ; \\
{ }^{A B} \nabla_{b}^{(-\alpha,-n)} f(t) & =\left({ }^{A B R} \nabla_{b}^{\alpha}\right)^{n} f(t) .
\end{aligned}
$$

For a proof of this, see Theorem 2 below.

- In the case $\alpha \rightarrow 1$, we have the following conventions:

$$
\begin{aligned}
{ }_{a}^{A B} \nabla^{(-1, \mu)} f(t) & =\left({ }_{a} \nabla^{-1} f(t)\right)^{\mu}={ }_{a} \nabla^{-\mu} f(t) ; \\
{ }_{a}^{A B} \nabla^{(-1,-\mu)} f(t) & ={ }_{a} \nabla^{\mu} f(t) .
\end{aligned}
$$

Theorem 2. For any $a<b$ in $\mathbb{R}, \alpha \in(0,1)$, and $n \in \mathbb{N}$, the identities (21) and (22) are valid.

Proof. We prove the result in the special case $n=1$, i.e., Equations (19) and (20). The general result follows from these two equations together with the semigroup property proven in the next section (Theorem 4). By symmetry, we consider only (19).

Substituting $\mu=1$ in the representation (18), we find:

$$
{ }_{a}^{A B} \nabla^{(-\alpha,-1)} f(t)=\sum_{k=0}^{\infty} \frac{\left(\begin{array}{c}
-1 \\
k
\end{array}\right) B(\alpha)^{1} \alpha^{k}}{(1-\alpha)^{1+k}}{ }_{a} \nabla^{-k \alpha} f(t) .
$$

Since we know that $\left(\begin{array}{c}-1 \\ k\end{array}\right)=(-1)^{k}$ and ${ }_{a} \nabla^{-0} f(t)=f(t)$, this expression becomes:

$$
{ }_{a}^{A B} \nabla^{(-\alpha,-1)} f(t)=\frac{B(\alpha)}{1-\alpha}\left[f(t)+\sum_{k=1}^{\infty}\left(\frac{-\alpha}{1-\alpha}\right)^{k}\left({ }_{a} \nabla^{-\alpha k} f\right)(t)\right] .
$$

Lemma 6 tells us that the right-hand side of the final equation is precisely ${ }_{a}^{A B R} \nabla^{\alpha} f(t)$, as required.

Example 1. As an illustrative example, we apply the operators introduced in Definitions 8 and 9 to some simple functions, as follows:

$$
\begin{aligned}
{ }_{a}^{A B} \nabla^{(-\alpha, \mu)}(t-a)^{\overline{\gamma-1}} & =\sum_{k=0}^{\infty} \frac{\left(\begin{array}{l}
\mu \\
k
\end{array}\right)(1-\alpha)^{\mu-k} \alpha^{k}}{B(\alpha)^{\mu}}{ }_{a} \nabla^{-k \alpha}(t-a)^{\overline{\gamma-1}} \\
& =\sum_{k=0}^{\infty} \frac{\left(\begin{array}{l}
\mu \\
k
\end{array}\right)(1-\alpha)^{\mu-k} \alpha^{k}}{B(\alpha)^{\mu}} \cdot \frac{\Gamma(\gamma)}{\Gamma(\gamma+k \alpha)}(t-a)^{\overline{\gamma+k \alpha-1}} \\
{ }^{A B} \nabla_{b}^{(-\alpha, \mu)}(b-t)^{\overline{\gamma-1}} & =\sum_{k=0}^{\infty} \frac{\left(\begin{array}{l}
\mu \\
k
\end{array}\right)(1-\alpha)^{\mu-k} \alpha^{k}}{B(\alpha)^{\mu}} \nabla_{b}^{-k \alpha}(b-t)^{\overline{\gamma-1}} \\
& =\sum_{k=0}^{\infty} \frac{\left(\begin{array}{l}
\mu \\
k
\end{array}\right)(1-\alpha)^{\mu-k} \alpha^{k}}{B(\alpha)^{\mu}} \cdot \frac{\Gamma(\gamma)}{\Gamma(\gamma+k \alpha)}(b-t)^{\overline{\gamma+k \alpha-1}} .
\end{aligned}
$$

Note that we have used here the following facts ([24] Lemma 3.3, and [23] Proposition 3.8):

$$
\begin{aligned}
{ }_{a} \nabla^{-\alpha}(t-a)^{\overline{\gamma-1}} & =\frac{\Gamma(\gamma)}{\Gamma(\gamma+\alpha)}(t-a)^{\overline{\alpha+\gamma-1}} \\
\nabla_{b}^{-\alpha}(b-t)^{\overline{\gamma-1}} & =\frac{\Gamma(\gamma)}{\Gamma(\gamma+\alpha)}(b-t)^{\overline{\gamma+\alpha-1}}
\end{aligned}
$$




\subsection{Fundamental Properties}

In this section, we prove some important properties of the definition proposed in the previous section, which demonstrate its naturality and usefulness.

Theorem 3 (Nabla discrete Laplace transforms in the iterated AB model). Let $\alpha, \mu$, and $f$ be as in Definition 8. Then, we have:

$$
\left(\mathcal{K}_{a}{ }_{a}^{A B} \nabla^{(-\alpha, \mu)} f\right)(z)=\left(\frac{1-\alpha}{B(\alpha)}+\frac{\alpha}{B(\alpha)} z^{-\alpha}\right)^{\mu}\left(\mathcal{K}_{a} f\right)(z)
$$

Proof. We use the Definition 8 of the iterated AB fractional difference and Lemma 4 concerning the nabla discrete Laplace transform of fractional difference operators.

$$
\begin{aligned}
\left(\mathcal{K}_{a}{ }_{a}^{A B} \nabla^{(-\alpha, \mu)} f\right)(z) & =\sum_{k=0}^{\infty} \frac{\left(\begin{array}{l}
\mu \\
k
\end{array}\right)(1-\alpha)^{\mu-k} \alpha^{k}}{B(\alpha)^{\mu}}\left(\mathcal{K}_{a} \nabla^{-k \alpha} f\right)(z) \\
& =\sum_{k=0}^{\infty} \frac{\left(\begin{array}{l}
\mu \\
k
\end{array}\right)(1-\alpha)^{\mu-k} \alpha^{k}}{B(\alpha)^{\mu}} z^{-\alpha k}\left(\mathcal{K}_{a} f\right)(z) \\
& =\sum_{k=0}^{\infty} \frac{\left(\begin{array}{l}
\mu \\
k
\end{array}\right)(1-\alpha)^{\mu-k}\left(z^{-\alpha} \alpha\right)^{k}}{B(\alpha)^{\mu}}\left(\mathcal{K}_{a} f\right)(z) \\
& =\left(\frac{1-\alpha}{B(\alpha)}+\frac{\alpha}{B(\alpha)} z^{-\alpha}\right)^{\mu}\left(\mathcal{K}_{a} f\right)(z),
\end{aligned}
$$

where in the last step, the binomial theorem is applied.

Theorem 4 (The semigroup property). Let $\alpha \in[0,1], \mu, v \in \mathbb{R}$, and $f$ be a function defined on $\mathbb{N}_{a}$. Then, the left and right iterated $A B$ fractional difference operators each have the semigroup property in $\mu$. Namely, for any $t \in \mathbb{N}_{a}$, we have:

$$
{ }_{a}^{A B} \nabla^{(-\alpha, \mu)}{ }_{a}^{A B} \nabla^{(-\alpha, v)} f(t)={ }_{a}^{A B} \nabla^{(-\alpha,(\mu+v))} f(t),
$$

and for any $t \in{ }_{b} \mathbb{N}$, we have:

$$
{ }^{A B} \nabla_{b}^{(-\alpha, \mu)}{ }^{A B} \nabla_{b}^{(-\alpha, v)} f(t)={ }^{A B} \nabla_{b}^{(-\alpha,(\mu+v))} f(t),
$$

Proof. Using the fact (6) that the left fractional sums have the semigroup property and by the help of the identity

$$
\sum_{k=0}^{m}\left(\begin{array}{l}
\mu \\
k
\end{array}\right)\left(\begin{array}{c}
v \\
m-k
\end{array}\right)=\left(\begin{array}{c}
\mu+v \\
m
\end{array}\right)
$$

we have:

$$
\begin{aligned}
& { }_{a}^{A B} \nabla^{(-\alpha, \mu)}{ }_{a}^{A B} \nabla^{(-\alpha, v)} f(t) \\
& =\sum_{k=0}^{\infty} \frac{\left(\begin{array}{l}
\mu \\
k
\end{array}\right)(1-\alpha)^{\mu-k} \alpha^{k}}{B(\alpha)^{\mu}}{ }_{a} \nabla^{-k \alpha}\left[\sum_{n=0}^{\infty} \frac{\left(\begin{array}{l}
v \\
n
\end{array}\right)(1-\alpha)^{v-n} \alpha^{n}}{B(\alpha)^{v}}{ }_{a} \nabla^{-n \alpha} f(t)\right] \\
& =\sum_{k, n} \frac{\left(\begin{array}{l}
\mu \\
k
\end{array}\right)\left(\begin{array}{l}
v \\
n
\end{array}\right)(1-\alpha)^{\mu+v-k-n} \alpha^{k+n}}{B(\alpha)^{\mu+v}}{ }_{a} \nabla^{-k \alpha}{ }_{a} \nabla^{-n \alpha} f(t) \\
& =\sum_{m=0}^{\infty} \sum_{k=0}^{m} \frac{\left(\begin{array}{l}
\mu \\
k
\end{array}\right)\left(\begin{array}{c}
v \\
m-k
\end{array}\right)(1-\alpha)^{\mu+v-m} \alpha^{m}}{B(\alpha)^{\mu+v}}{ }_{a} \nabla^{-m \alpha} f(t) \\
& =\sum_{m=0}^{\infty} \frac{\left(\begin{array}{c}
\mu+v \\
m
\end{array}\right)(1-\alpha)^{\mu+v-m} \alpha^{m}}{B(\alpha)^{\mu+v}}{ }_{a} \nabla^{-m \alpha} f(t)={ }_{a}^{A B} \nabla^{(-\alpha, \mu+v)} f(t) .
\end{aligned}
$$


This proves the left semigroup property (24). The proof for (25) is similar, starting from (7) and using the same binomial identity.

Theorem 5 (Integration by parts). Let $\alpha \in[0,1], \mu \in \mathbb{R}$, and $a, b \in \mathbb{R}$ with $a \equiv b$ modulo one. For any function $f$ defined on $\mathbb{N}_{a}$ and $g$ defined on ${ }_{b} \mathbb{N}$, we have the following integration by parts identity:

$$
\sum_{s=a+1}^{b-1} g(s){ }_{a}^{A B} \nabla^{(-\alpha, \mu)} f(s)=\sum_{s=a+1}^{b-1} f(s)^{A B} \nabla_{b}^{(-\alpha, \mu)} g(s) .
$$

Proof. We know from [24] (Theorem 4.1) the following integration by parts identity for standard fractional difference-sum operators:

$$
\sum_{s=a+1}^{b-1} g(s)_{a} \nabla^{-\alpha} f(s)=\sum_{s=a+1}^{b-1} f(s) \nabla_{b}^{-\alpha} g(s) .
$$

Using respectively Definition 8, Equation (27), and Definition 9, we have:

$$
\begin{aligned}
\sum_{s=a+1}^{b-1} g(s){ }_{a}^{A B} \nabla^{(-\alpha, \mu)} f(s) & =\sum_{s=a+1}^{b-1} g(s) \sum_{k=0}^{\infty} \frac{\left(\begin{array}{l}
\mu \\
k
\end{array}\right)(1-\alpha)^{\mu-k} \alpha^{k}}{B(\alpha)^{\mu}}{ }_{a} \nabla^{-k \alpha} f(s) \\
& =\sum_{k=0}^{\infty} \frac{\left(\begin{array}{l}
\mu \\
k
\end{array}\right)(1-\alpha)^{\mu-k} \alpha^{k}}{B(\alpha)^{\mu}} \sum_{s=a+1}^{b-1} g(s){ }_{a} \nabla^{-k \alpha} f(s) \\
& =\sum_{k=0}^{\infty} \frac{\left(\begin{array}{l}
\mu \\
k
\end{array}\right)(1-\alpha)^{\mu-k} \alpha^{k}}{B(\alpha)^{\mu}} \sum_{s=a+1}^{b-1} f(s) \nabla_{b}^{-k \alpha} g(s) \\
& =\sum_{s=a+1}^{b-1} f(s) \sum_{k=0}^{\infty} \frac{\left(\begin{array}{l}
\mu \\
k
\end{array}\right)(1-\alpha)^{\mu-k} \alpha^{k}}{B(\alpha)^{\mu}} \nabla_{b}^{-k \alpha} g(s) \\
& =\sum_{s=a+1}^{b-1} f(s)^{A B} \nabla_{b}^{(-\alpha, \mu)} g(s) .
\end{aligned}
$$

Integration by parts, the identities as in Theorem 5 are the main tool used to study discrete variational problems in the frame of iterated $A B$ difference-sums $[17,29]$.

\section{Fractional Difference Equations and Applications}

Let $\alpha \in(0,1]$ and $\mu \in \mathbb{R}^{+}$. Consider a general fractional ordinary difference equation of the form:

$$
{ }_{0}^{A B} \nabla^{(-\alpha,-\mu)} x(t)=-A x(t)+b(t), \quad A \in \mathbb{R}^{+} .
$$

We search for a series solution, i.e., one of the form:

$$
x(t)=\sum_{s=0}^{\infty} c_{s} \bar{t}^{\overline{\alpha s}},
$$

where we assume the given function $b$ can be written as:

$$
b(t)=\sum_{s=0}^{\infty} b_{s} t^{\overline{\alpha s}} .
$$

First, we substitute $x(t)$ into the left-hand side of (28) and utilise Lemma 1 to get: 


$$
\begin{aligned}
{ }_{0}^{A B} \nabla^{(-\alpha,-\mu)} x(t) & =\sum_{k=0}^{\infty} \frac{\left(\begin{array}{c}
-\mu \\
k
\end{array}\right)(1-\alpha)^{-\mu-k}}{B(\alpha)^{-\mu}}{ }_{0} \nabla^{-\alpha k}\left(\sum_{i=0}^{\infty} c_{i} t^{\overline{i \alpha}}\right) \\
& =\sum_{k=0}^{\infty} \sum_{i=0}^{\infty} \frac{\left(\begin{array}{c}
-\mu \\
k
\end{array}\right)(1-\alpha)^{-\mu-k}}{B(\alpha)^{-\mu}} c_{i} \frac{\Gamma(i \alpha+1)}{\Gamma((k+i) \alpha+1)} t^{\overline{(i+k) \alpha}} \\
& =\sum_{m=0}^{\infty} \frac{t^{\overline{m \alpha}}}{B(\alpha)^{-\mu} \Gamma(m \alpha+1)} \sum_{k=0}^{m} c_{m-k}\left(\begin{array}{c}
-\mu \\
k
\end{array}\right)(1-\alpha)^{-\mu-k} \Gamma((m-k) \alpha+1),
\end{aligned}
$$

where we have set $m=k+i$ in the last line. On the other hand, the right-hand side of (28) can be written as:

$$
\sum_{m=0}^{\infty}\left[-A c_{m}+b_{m}\right] t^{\overline{\alpha m}}
$$

Now, if we equate the coefficients in these two infinite series, we reach the identity:

$$
\frac{1}{\Gamma(m \alpha+1)} \sum_{k=0}^{m} \frac{c_{m-k}\left(\begin{array}{c}
-\mu \\
k
\end{array}\right) B(\alpha)^{\mu} \alpha^{k} \Gamma((m-k) \alpha+1)}{(1-\alpha)^{\mu+k}}=-A c_{m}+b_{m}, \quad m \in \mathbb{N}_{0} .
$$

Solving for $m=0$, we have:

$$
c_{0}=\frac{b_{0}}{A+\left(\frac{B(\alpha)}{1-\alpha}\right)^{\mu}}
$$

For positive $m$, the identity (29) will yield:

$$
c_{m}=\frac{b_{m}}{A+\left(\frac{B(\alpha)}{1-\alpha}\right)^{\mu}}-\sum_{k=1}^{\infty} \frac{c_{m-k}\left(\begin{array}{c}
-\mu \\
k
\end{array}\right) \alpha^{k} B(\alpha)^{\mu} \Gamma((m-k) \alpha+1)}{(1-\alpha)^{\mu+k} \Gamma(m \alpha+1) \Gamma(m \alpha+1)\left(A+\left(\frac{B(\alpha)}{1-\alpha}\right)^{\mu}\right)} .
$$

Hence, we obtain the following solution:

$$
x(t)=\frac{b(t)}{A+\left(\frac{B(\alpha)}{1-\alpha}\right)^{\mu}}-\sum_{m=1}^{\infty} t^{\overline{m \alpha}} \sum_{k=1}^{m} \frac{c_{m-k}\left(\begin{array}{c}
-\mu \\
k
\end{array}\right) \alpha^{k} B(\alpha)^{\mu} \Gamma((m-k) \alpha+1)}{(1-\alpha)^{\mu+k} \Gamma(m \alpha+1)\left(A+\left(\frac{B(\alpha)}{1-\alpha}\right)^{\mu}\right)} .
$$

Actually, the coefficients $c_{i}, i=1,2,3, \ldots$ can be calculated recursively from (30).

Remark 3. Of special interest is the particular case $\mu=1$ of the above problem. In this case, the solution representation (31) becomes:

$$
x(t)=\frac{b(t)}{A+\left(\frac{B(\alpha)}{1-\alpha}\right)}-\sum_{m=1}^{\infty} t^{\overline{m \alpha}} \sum_{k=1}^{m} \frac{c_{m-k}(-1)^{k} \alpha^{k} B(\alpha) \Gamma((m-k) \alpha+1)}{(1-\alpha)^{1+k} \Gamma(m \alpha+1)\left(A+\frac{B(\alpha)}{1-\alpha}\right)},
$$

this being a solution of the fractional difference equation:

$$
{ }_{0}^{A B R} \nabla^{\alpha} x(t)=-A x(t)+b(t),
$$

where $b(t)=\sum_{s=0}^{\infty} b_{s} t^{\overline{\alpha s}}$ and $c_{0}=\frac{b_{0}}{A+\left(\frac{B(\alpha)}{1-\alpha}\right)}$ and the coefficients $c_{i}, i \in \mathbb{N}$, can be determined from (30) with $\mu=1$.

Remark 4. It is worth noting that the semigroup property of Theorem 4 will be invaluable in the further study of fractional difference equations in the discrete iterated $A B$ model. There are many classes of difference equations which are easy to solve when we have a semigroup property, but difficult or impossible when we do not $[2,22,23]$. 
Equipped with a semigroup property, we can easily cancel operators, in order to simplify and solve an equation, by applying new difference-sum operators to both the left and right sides of the equation.

\section{Conclusions}

In this paper, we introduced a new kind of discrete fractional calculus, whose advantages over existing models can be summarised as follows.

- Atangana-Baleanu fractional calculus, in the continuous case, has discovered many applications in modelling fractional systems with non-local and non-singular dynamics, behaviour that cannot be modelled using the classical Riemann-Liouville kernels. In a short space of time, the AB formula has established itself as a major competitor among the many different approaches to fractional calculus.

- Discrete fractional calculus (DFC) is a whole different field of research from continuous fractional calculus. Like the original discrete calculus and difference equations, DFC can be used to study many real-world processes whose behaviour is too discrete to be well modelled by continuous fractional calculus.

- In any type of calculus-discrete or continuous, integer-order or fractional—an important question is whether or not a semigroup property is satisfied. If we apply the operator twice with order $\alpha$, do we get the same result as by applying it once with order $2 \alpha$ ? This fundamental issue has given rise to much debate about the validity of certain fractional models, and several new models have been proposed purely in order to regain a semigroup property.

Our definition gives a unique way of combining the particular structure of the AB formula, the discrete behaviour of DFC, and the semigroup property thanks to the introduction of a second parameter. Previous literature covered the combination of any two of these three (the discrete AB model, the continuous iterated AB model, and discrete models with semigroup properties), but this is the first time that all three have been put together.

In this paper, we covered some basic properties and examples of our new fractional difference-sum operator. By analysing the effect on it of the discrete Laplace transform, we also demonstrated how it can be used to solve a certain family of fractional difference equations.

Numerical methods have been an important part of the recent development of fractional calculus, including in the Atangana-Baleanu model [6,15]. Discrete calculus is often better suited to numerical schemes than the continuous case, due to the finite structure of computation there [5]. Thus, we expect that any new progress in discrete fractional calculus of $A B$ type should have ramifications in numerical analysis, although exploring such ramifications would be beyond the scope of the current paper.

Author Contributions: Conceptualisation, T.A. and A.F.; formal analysis, T.A. and A.F.; writing, T.A. and A.F.

Funding: The first author would like to thank Prince Sultan University for funding this work through research group Nonlinear Analysis Methods in Applied Mathematics (NAMAM) Group Number RG-DES-2017-01-17. The second author would like to thank the Engineering and Physical Sciences Research Council (EPSRC) for their support in the form of a research student grant.

Conflicts of Interest: The authors declare no conflict of interest.

\section{References}

1. Miller, K.S.; Ross, B. Fractional difference calculus. In Proceedings of the International Symposium on Univalent Functions, Fractional Calculus and Their Applications; Nihon University: Koriyama, Japan, 1989; pp. 139-152.

2. Goodrich, C.; Peterson, A. Discrete Fractional Calculus; Springer: London, UK, 2015.

3. Holm, M. The Theory of Discrete Fractional Calculus: Development and Application. Ph.D. Thesis, University of Nebraska, Lincoln, NE, USA, 2011.

4. Bohner, M.; Peterson, A. Advances in Dynamic Equations on Time Scales; Birkhäuser, Boston-Basel-Berlin: Basel, Switzerland, 2003. 
5. Lakshmikantham, V.; Trigiante, D. Theory of Difference Equations: Numerical Methods and Applications; Marcel Dekker: New York, NY, USA, 2002.

6. Uçar, S.; Uçar, E.; Özdemir, N.; Hammouch, Z. Mathematical analysis and numerical simulation for a smoking model with Atangana-Baleanu derivative. Chaos Solitons Fractals 2019, 118, 300-306. [CrossRef]

7. Yusuf, A.; Qureshi, S.; Inc, M.; Aliyu, A.I.; Baleanu, D.; Shaikh, A.A. Two-strain epidemic model involving fractional derivative with Mittag-Leffler kernel. Chaos 2018, 28, 123121. [CrossRef] [PubMed]

8. Jajarmi, A.; Baleanu, D. Suboptimal control of fractional-order dynamic systems with delay argument. J. Vib. Control 2018, 24, 2430-2446. [CrossRef]

9. Baleanu, D.; Machado, J.A.T.; Luo, A.C.J. Fractional Dynamics and Control; Springer Science and Business Media: London, UK, 2012.

10. Magin, R.L. Fractional Calculus in Bioengineering; Begell House Publishers: Danbury, CT, USA, 2006.

11. Bonfanti, A.; Fouchard, J.; Khalilgharibi, N.; Charras, G.; Kabla, A. A unified rheological model for cells and cellularised materials. bioRxiv 2019. [CrossRef]

12. Caputo, M.; Fabrizio, M. A new definition of fractional derivative without singular kernel. Prog. Fract. Differ. Appl. 2015, 1, 73-85.

13. Atangana, A.; Baleanu, D. New fractional derivative with non-local and non-singular kernel. Therm. Sci. 2016, 20, 757-763. [CrossRef]

14. Baleanu, D.; Fernandez, A. On some new properties of fractional derivatives with Mittag-Leffler kernel. Commun. Nonlinear Sci. Numer. Simul. 2018, 59, 444-462. [CrossRef]

15. Djida, J.-D.; Atangana, A.; Area, I. Numerical Computation of a Fractional Derivative with Non-Local and Non-Singular Kernel. Math. Model. Nat. Phenom. 2017, 12, 4-13. [CrossRef]

16. Abdeljawad, T.; Al-Mdallal, Q.M. Discrete Mittag-Leffler kernel type fractional difference initial value problems and Gronwall's inequality. J. Comp. Appl. Math. 2018, 339, 218-230. [CrossRef]

17. Abdeljawad, T.; Baleanu, D. Discrete fractional differences with nonsigular discrete Mittag-Leffler kernels. Adv. Differ. Equ. 2016, 2016, 232. [CrossRef]

18. Fernandez, A.; Baleanu, D. On a new definition of fractional differintegrals with Mittag-Leffler kernel. arXiv 2018, arXiv:1807.10601.

19. Fernandez, A.; Baleanu, D.; Srivastava, H.M. Series representations for models of fractional calculus involving generalised Mittag-Leffler functions. Commun. Nonlinear Sci. Numer. Simul. 2019, 67, 517-527. [CrossRef]

20. Atici, F.M.; Uyanik, M. Analysis of Discrete Fractional Operators. Appl. Anal. Discret. Math. 2015, 9, 139-149. [CrossRef]

21. Herrmann, R. Fractional Calculus: An Introduction for Physicists, 2nd ed.; World Scientific: Singapore, 2014.

22. Abdeljawad, T. On Delta and Nabla Caputo Fractional Differences and Dual Identities. Discret. Dyn. Nat. Soc. 2013, 2013, 406910. [CrossRef]

23. Abdeljawad, T. Dual identities in fractional difference calculus within Riemann. Adv. Differ. Equ. 2013, 2013, 36. [CrossRef]

24. Abdeljawad, T.; Atici, F. On the definitions of nabla fractional differences. Abstr. Appl. Anal. 2012, 2012, 406757. [CrossRef]

25. Atıc1, F.M.; Eloe, P.W. Discrete fractional calculus with the nabla operator. Electron. J. Qual. Theory Differ. Equ. 2009, 2009, 1-12. [CrossRef]

26. Abdeljawad, T.; Jarad, F.; Baleanu, D. A semigroup-like property for discrete Mittag-Leffler functions. Adv. Differ. Equ. 2012, 1, 72. [CrossRef]

27. Mainardi, F. On some properties of the Mittag-Leffler function $E_{\alpha}\left(-t^{\alpha}\right)$ completely monotone for $t>0$ with $0<\alpha<1$. Discret. Contin. Dyn. Syst. -Ser. B (DCDS-B) 2014, 19, 2267-2278.

28. Kilbas, A.A.; Saigo, M.; Saxena, K. Generalized Mittag-Leffler function and generalized fractional calculus operators. Integral Transform. Spec. Funct. 2004, 15, 31-49. [CrossRef]

29. Abdeljawad, T. Nabla Euler-Lagrange equations in discrete fractional variational calculus within Riemann and Caputo. Int. J. Math. Comput. 2014, 22, 144-153.

(C) 2019 by the authors. Licensee MDPI, Basel, Switzerland. This article is an open access article distributed under the terms and conditions of the Creative Commons Attribution (CC BY) license (http:/ / creativecommons.org/licenses/by/4.0/). 